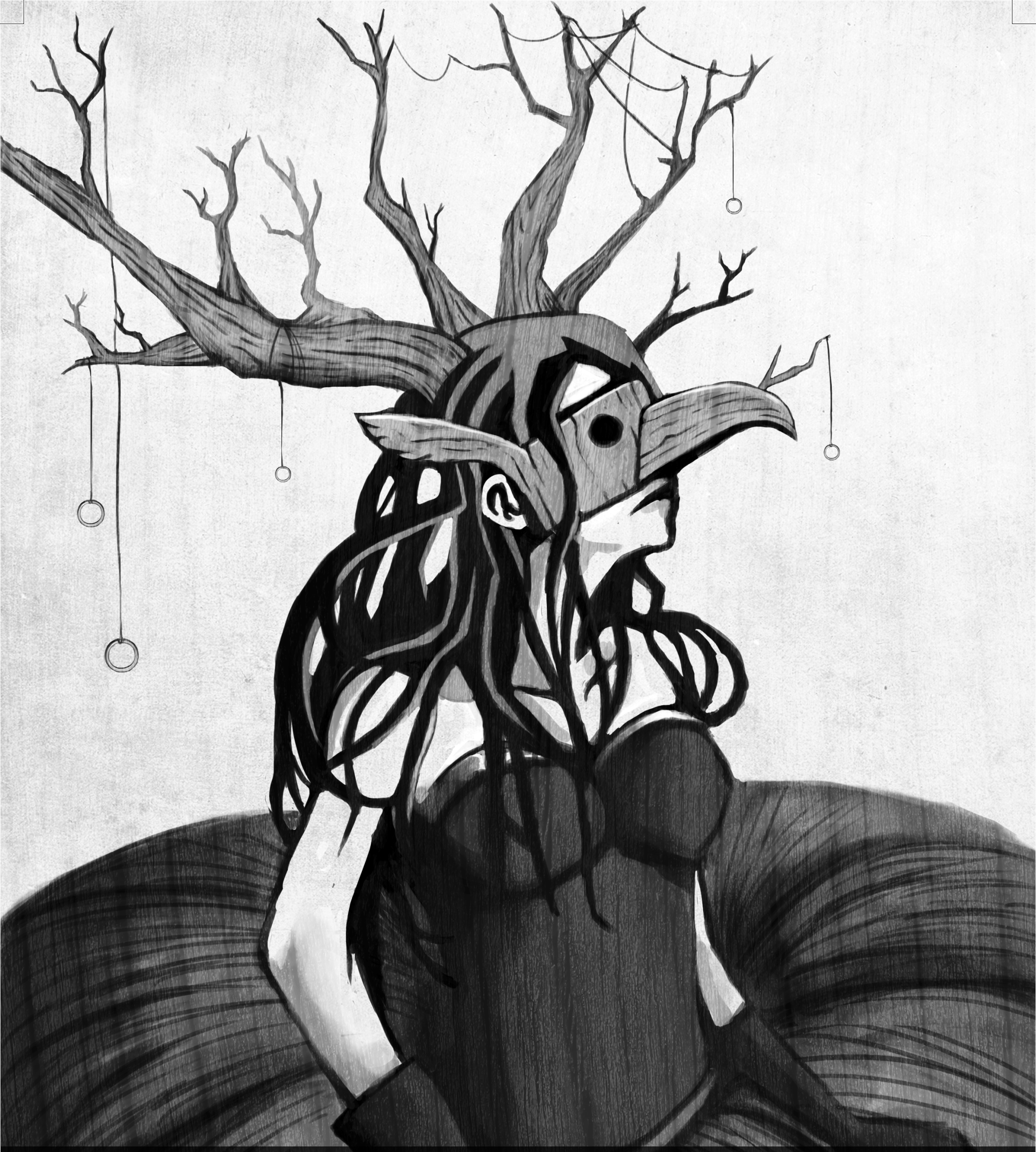

Exploración de una estrategia para la enseñanza en un curso de procesos básicos del programa de Psicología de una universioad privada de Bogotá D. C. 


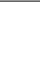




\section{Exploración de una estrategia para la enseñanza en un curso de procesos básicos del programa de Psicología de una universidad privada de Bogotá D. C. ${ }^{1}$}

\author{
Nicolás Arias Velandia
}

nariasv@gmail.com

Universidad Nacional de Colombia
Fecha de recepción: 18 de julio de 2011

Fecha de aprobación: 30 de septiembre de 2011

\section{Resumen}

Este estudio exploró los efectos de dos unidades temáticas de un curso de procesos básicos en psicología, en los cuales se desarrolló con los estudiantes la formulación de un problema de investigación y su resolución mediante un experimento. Para ello se implementaron dos ciclos basados en teorías de solución de pro-blemas, de aprendizaje basado en problemas, y de investigación del profesor en el aula. Los resultados mostraron que los estudiantes aprendieron la mecánica de la formulación del problema de investigación y conceptos relacionados con el mismo, con poco avance en la elaboración de informes de laboratorio y en comprensión de la lógica de cambio del conocimiento científico. Se discuten los alcances y las limitaciones de la experiencia desarrollada.

\section{Palabras clave}

Solución de problemas, análisis de tareas, aprendizaje basado en problemas, investigación en el aula, profesor - investigador, aprendizaje de las ciencias.

\section{Abstract}

This study explored the effect of two thematic units taught in a basic processes course in psychology, in which the professor and the students developed a research problem and solved it using an experiment. It was deve-loped through two cycles based in problem solving theories, in problem-based learning and in professor / teacher research in classroom. Results showed that students learned the mechanisms to formulate a research problem and concepts related to this problem, but their learning was less in making laboratory reports and in comprehension of logics involved in scientific knowledge change. In this work we discuss the ranges and limitations of the experience.

\section{Keywords}

Problem Solving, Task Analysis, Problem Based Learning, Research in Classroom, Professor Researcher, Science Learning.

1 Este artículo reporta los resultados de una investigación participante realizada por iniciativa del autor sin participación de entidades patrocinadoras o financiadoras. El autor agradece la colaboración en infraestructura y desarrollo al Laboratorio de Psicología, en cabeza del profesor Jaime Castro Martínez y al Departamento Académico de Psicología, en cabeza de Rodrigo Riaño Pineda, de Politécnico Grancolombiano Institución Universitaria. Se agradece también la colaboración a Billy Escobar, decano de la Facultad de Ciencias Sociales de la misma institución.

Se agradece también a María Cristina Torres Pedraza, coordinadora pedagógica de la Fundación Universitaria Sanitas y estudiante de Maestría en Pedagogía de la Universidad de La Sabana, por el acceso a bibliografía sobre aprendizaje basado en problemas. 


\section{Introducción}

El desarrollo de una clase está mediado por tres grandes componentes: el acervo del estudiante, el acervo del profesor y el tipo de actividades que se dan al estudiante a desarrollar para que logre su aprendizaje (Florez, Castro \& Arias, 2009). Sin embargo, estos factores se desarrollan en el contexto de un entorno institucional con una cultura propia o curriculo, que hace que sus estudiantes y profesores adquieran también unas características particulares y que valoren ciertos aspectos, lo cual incide en el aprendizaje que finalmente logran los estudiantes (Rodriguez, 2007). En el caso de la educación superior, postsecundaria o universitaria, este contexto parece cobrar un peso aún mayor.

Este estudio explora los alcances, efectos y limitaciones de una estrategia implementada en la enseñanza en un curso de procesos básicos en psicología en una universidad, con base en dos unidades temáticas con respecto al acervo de los estudiantes, el acervo del profesor, el tipo de actividades propuestas y el contexto institucional en el curso universitario en el cual se desarrolla.

Se asume que el aprendizaje de los estudiantes de los contenidos de un curso universitario debe contar con dos elementos: el aporte del profesor y el aporte del estudiante (Duenas, 2001; Echavarria, 2010; Gorbaneff \& Cancino, 2009; Kindler, Grant, Kulla, Poole \& Godolphin, 2009; Kreiner, 2009; Newton $\&$ Wood, 2009; Restrepo Gómez, 2005). Se espera que el estudiante tenga oportunidad de ejercitar sus habilidades y comprender los conceptos subyacentes a dichas habilidades. Y además se espera que el acervo del profesor, las actividades que se desarrollan en clase y el contexto institucional, creen situaciones favorables para que el estudiante tenga estas oportunidades de trabajo y haga uso efectivo de las mismas (Restrepo Gómez, 2005).

De otra parte, se espera que el profesor sea una persona que domina conocimientos convencionales o propios de la disciplina que dicta y que además medie entre el conocimiento previo de los estudiantes y el conocimiento que se busca que adquieran o dominen (Vygotski, 1978; Rodríguez, 2007). En ese sentido, se busca que con ayuda de esta destreza, el docente cree situaciones con propósito definido de aprendizaje que lleven a los estudiantes de su conocimiento previo a conocimientos convencionales, en un área particular de su disciplina, con algunos alcances y limitaciones dado el conocimiento previo de los estudiantes y el valor o centralidad que tenga el área impartida dentro de la estructura curricular del plan de formación del estudiante (Rodríguez, 2007).

En sintesis, una buena descripción del fin buscado en una clase de nivel universitario es hacer que el estudiante pase de ser un conocedor en habilidades académicas básicas, a ser una persona con conocimiento consolidado de habilidades y conceptos básicos de su disciplina (Carlino, 2005; Flórez \& Gutiérrez, 2009). Se busca entonces que el estudiante pase a ser parte del entorno social y de la tradición de saber propia de la disciplina que estudia, a medida que va adquiriendo responsabilidades mayores en el curso de su formación.

En la búsqueda de esta gran meta surgen varias inquietudes: ¿cómo hacer para que los estudiantes realmente comprendan los contenidos y no solamente los repitan?, ¿cómo lograr que de las actividades de enseñanza se consoliden conocimientos y no solamente se tomen apuntes que se utilizarán exclusivamente para los exámenes?, ¿cómo llegaremos a hacer que aquello que los estudiantes repasan, lo utilicen como parte de su acervo de conocimientos y habilidades para enfrentar situaciones, incluso cuando no son similares a las que se utilizaron en las situaciones de aprendizaje?

Este artículo no pretende dar respuesta a todas estar preguntas. En lugar de eso, muestra cómo se desarrolló una estrategia para la enseñanza en un curso de procesos básicos en psicología ${ }^{2}$ en una universidad privada de Bogotá, Colombia, partiendo de las siguientes orientaciones teóricas para su desarrollo:

\section{El aprendizaje efectivo en los cursos univer- sitarios se logra mediante situaciones}

2 Generalmente, se considera en la formación en cursos universitarios de pregrado en psicología, que hay un subconjunto de los mismos que son sobre procesos básicos como percepción, cognición, emoción, aprendizaje, motivación u otros similares, que imparten conocimientos sobre los mecanismos básicos de funcionamiento psicológico de las personas y de los organismos. Generalmente estos cursos presentan una dificultad para los estudiantes: no parecen tener para ellos alguna utilidad práctica que sea evidente a primera vista. Por este motivo, es frecuente que dichos cursos necesiten arreglos didácticos para hacerlos más relevantes para el punto de vista del alumno. 
estructuradas. Las situaciones de aprendizaje deben siempre obedecer a un propósito formativo, a un objetivo de aprendizaje, y debe evaluarse hasta qué punto se pueden convertir en situaciones meramente rutinarias o repetitivas sin una meta clara (Ministerio de Educacion Nacional, Colombia, 2009).

2. El aprendizaje efectivo en los cursos universitarios se logra mediante situaciones $y$ actividades en las cuales la meta es clara para el estudiante. Parte del trabajo a realizar para que una situación de aprendizaje sea efectiva es que se logre que el estudiante comprenda en cada momento qué sentido tiene cada actividad para su aprendizaje y para su desempeño esperado en el futuro (Duenas, 2001; Echavarria, 2010; Gorbaneff \& Cancino, 2009; Kindler et.al., 2009; Kreiner, 2009; Newton \& Wood, 2009; Restrepo Gomez, 2005).

3. El aprendizaje efectivo en los cursos universitarios requiere que se diseñen situaciones que pueden ser diferentes en cada disciplina. O podría decirse de otra manera: cada disciplina tiene unas formas particulares de proceder, de construir su saber y de comunicación entre sus especialistas y entre estos y el público general (Carlino, 2005). Por lo tanto, si parte de la enseñanza es crear situaciones en las cuales el estudiante conoce estas particularidades en su disciplina, la forma de enseñanza de las disciplinas debe ser cercana a esa tradición de saber que le es propia y particular (Carlino, 2005; Flórez \& Gutiérrez, 2009).

4. El aprendizaje efectivo en los cursos universitarios se logra mediante situaciones que requieran de la utilización de los conceptos propios del área que se está comprendiendo. Como se plantea desde algunos paradigmas de estudio del aprendizaje en contexto avanzado, como el Aprendizaje Basado en Problemas (ABP), el aprendizaje debe estar orientado a que los estudiantes alcancen una meta para la cual deberán utilizar y desarrollar conceptos propios de la disciplina en la cual se forman. Esto requiere entonces un cuidadoso diseño de las situaciones que se utilizan para el desarrollo de una clase (Duenas, 2001; Echavarria, 2010; Gorbaneff \& Cancino, 2009; Kindler et.al., 2009; Kreiner, 2009; Newton \& Wood, 2009; Restrepo Gómez, 2005).
5. El aprendizaje efectivo en los cursos universitarios se logra mediante situaciones que muestren al estudiante la forma en la que los expertos suelen desarrollar su trabajo y la manera de resolverlo. Parte del aprendizaje de los estudiantes está en mostrarles una manera efectiva en la cual realiza su trabajo el profesor, para tener un modelo claro y una visualización de cómo proceder ante situaciones similares (Flórez, Arias \& Guzmán, 2006; Rojas \& Flórez, 2010; Larreamendy, 2003).

6. El aprendizaje efectivo en los cursos universitarios descansa en el conocimiento acumulado sobre el oficio de dictar clase en su temática especifica que han desarrollado los profesores. En el mundo educativo existen modelos conceptuales abstractos, modelos de situación, estrategias a implementar, etc., pero estas no existen fuera del contexto, diseño y situación específica que cada profesor enfrenta con cada curso en concreto. Por lo tanto, el saber acumulado que el profesor ha logrado sobre la enseñanza a través de las mismas actividades de enseñanza, le dan orientaciones sobre qué es acertado para desarrollar su actividad con cada curso particular (Stenhouse, 1980, 1981; Elliott, 2000).

Teniendo en cuenta estas orientaciones teóricas, se planteó un curso de Cognición y Lenguaje para un programa de pregrado de psicologia basado en laboratorios de investigación. Los laboratorios de investigación fueron situaciones que contaban con los siguientes elementos:

1. El planteamiento de una meta inicial por el profesor y de los pasos intermedios para lograrla, con sus requerimientos especificos en cada etapa (orientaciones teóricas $1,2,3,6)$.

2. La exposición magistral de conceptos teóricos que podrían ser necesarios para el desarrollo de un mini-problema de investigación en diferentes grupos (orientaciones teóricas $1,3,4,6)$.

3. Una exploración de dos o tres artículos de investigación relacionados con un problema de investigación escogido, de una gama de opciones dada previamente por el 
profesor (orientaciones teóricas 1, 2, 3, 4, 5).

4. Un ejercicio de deducción de un problema de investigación nuevo con los artículos escogidos previamente (orientaciones teóricas $2,3,5)$.

5. Un ejercicio de formulación de un método experimental para resolver el problema de investigación del punto anterior. Dicha formulación incluyó la descripción del plan experimental o diseño, características de los participantes, características de los instrumentos o tareas utilizadas y descripción detallada del procedimiento (orientaciones teóricas 2, 3, 5).

6. Un ejercicio empírico de aplicación del método experimental diseñado con niños o con adultos (según la unidad temática abordada en el curso) (orientaciones teóricas 2,5$)$.

7. Un ejercicio de escritura del reporte de investigación de cada sesión (orientaciones teóricas 2,5 ).

Estos mismos elementos se realizaron dos veces durante el curso: una vez en una unidad temática de cognición y lenguaje en adultos y otra en una unidad temática de cognición y lenguaje en niños. A continuación se describen las características metodológicas de este estudio, que consistió en la aplicación de los laboratorios descritos en los puntos anteriores.

\section{Método}

\section{Tipo de estudio y diseño}

Este es un estudio de orientación interpretativa o histórica-hermenéutica según la clasificación de Jurgen Habermas. Hace seguimiento a los desempeños de estudiantes en dos unidades temáticas de un curso de Cognición y Lenguaje (durante tres meses) de un programa de psicología de una universidad privada de Bogotá. El estudio utiliza técnicas de observación etnográfica participante, median-te un diario de trabajo del profesor (que es el mismo autor signatario de este artículo), que recopila tanto evidencias de observación del mismo profesor, como declaraciones verbales de los estudiantes sobre el desarrollo del curso; también utiliza datos cuantitativos tomados del programa y de los registros del docente.

\section{Participantes}

En el desarrollo del estudio participó un docente y sus dos cursos de la misma asignatura, uno en jornada diurna y otro en jornada nocturna. En el curso en jornada diurna participaron catorce estudiantes inscritos (diez mujeres y cuatro varones) y en el de jornada nocturna, 16 (quince mujeres y un varón). Todos eran estudiantes de cuarto semestre de psicología en una universidad privada de Bogotá.

\section{Procedimiento}

El trabajo implementado se desarrolló en dos unidades temáticas planteadas desde el inicio del curso por el profesor: una centrada en el trabajo experimental con adultos en el área de la asignatura y otra centrada en el trabajo experimental con niños. Ambas siguieron las siguientes fases de implementación:

Primera fase: compilación de conocimientos previos de los estudiantes y orientaciones básicas. En esta fase el profesor dijo a los estudiantes la meta final del trabajo en la unidad temática, sus pasos intermedios y las entregas parciales de productos para llegar a la meta. También realizó exposición magistral de algunos conceptos teóricos que se pueden utilizar en los experimentos y orientó la exploración y escogencia que hicieron los estudiantes de dos o tres artículos de investigación relacionados un problema escogido por ellos (tomado de una lista dada previamente antes de la unidad temática por el profesor).

Segunda fase: desarrollo del problema de investigación. En esta fase, el profesor partió de la exposición magistral de algunos conceptos teóricos que se pueden utilizar en los experimentos, dio orientaciones de procedimiento mostrando ejemplos al inicio de cada sesión y los estudiantes en grupos de trabajo de dos o tres personas realizaron la deducción del problema de investigación en los artículos y reportes, diseñaron el método para responder a la pregunta de investigación y realizaron un 
ejercicio de aplicación empírica del método experimental para responder a la pregunta de investigación. En la deducción del problema de investigación se pidió a los estudiantes que extrajeran aspectos comunes y diferentes en lo reportado en la teoría y en los resultados de los artículos escogidos para explicarles que de eso se trata un problema de investigación, mostrando que de ello se pueden extraer alcances y limitaciones de los estudios realizados. Luego de escribir esos alcances y limitaciones de los estudios (previa lectura de los mismos), los estudiantes procedieron a derivar de ello una pregunta que guiara su mismo trabajo y que superara los limites de las investigaciones reportadas en los artículos que leyeron. Esto se realizó en trabajo grupal en el aula de clases con asesorias del profesor a cada grupo de estudiantes. En sesiones siguientes se les pidió la formulación de un método para hacer un experimento, -vigilando que siempre cumpliera la característica de responder a la pregunta de investigación- y se planeó la realización efectiva del experimento. Según las facilidades, el experimento se realizó en el laboratorio de psicología de la universidad o se pidió videograbación para observar en una sesión cuando no pudiera ser revisado con el profesor en el equipo de video del laboratorio.

Tercera fase: realización de informe escrito del trabajo realizado. El profesor dictó a los estudiantes una serie de instrucciones para realizar el informe de investigación y mostró ejemplos de cada parte del informe y solicitó a los estudiantes que realizaran ejercicios de paso de sus apuntes al formato del informe para asegurar su comprensión. El formato de informe es una adaptación cercana al formato de artículos de investigación de la American Psychological Association (APA).

En todas las fases, el profesor pidió a los estudiantes que mostraran sus avances en cada una de las tareas requeridas y asignó una calificación general por todo este proceso. Al final calificó el informe de investigación presentado, tanto en su forma, como en su contenido. También recopiló notas del proceso a lo largo de todo el desarrollo de las dos unidades temáticas en la cuales se aplicó este procedimiento, recopiló datos cuantitativos del proceso de los estudiantes y al final recopiló datos generales de los mismos de los registros de la universidad.

\section{Resultados}

\section{Desarrollo de la estrategia de laboratorios}

En general, los estudiantes conocen conceptos básicos del área según lo que demuestran en el desarrollo del curso. Los estudiantes manifiestan ideas mucho más cercanas al conocimiento convencional en sus respuestas a los temas de los cuales trata el curso, como lo muestra la tabla 1 .

TABLA 1. NúMERO dE ESTUdiantes Y SUS TENDENCIAS DE RESPUESTA AL INICIO Y AL FINAL DE LAS DOS UNIDADES TEMÁTICAS (EN CURSIVA: RESPUESTAS CONVENCIONALES ESPERADAS, $\mathrm{N}=$ 30)

\begin{tabular}{lcc}
\hline $\begin{array}{l}\text { Núcleos de } \\
\text { respuestas }\end{array}$ & $\begin{array}{c}\text { Frecuencia al } \\
\text { inicio }\end{array}$ & $\begin{array}{c}\text { Frecuencia } \\
\text { al final }\end{array}$ \\
\hline $\begin{array}{l}\text { Procesos de } \\
\text { memoria }\end{array}$ & 13 & 2 \\
Pensamiento & 8 & 2 \\
$\begin{array}{l}\text { Información } \\
\text { Datos y } \\
\text { evidencias }\end{array}$ & 4 & 3 \\
$\begin{array}{l}\text { Lo que se } \\
\text { piensa y se } \\
\text { hace }\end{array}$ & 4 & \\
$\begin{array}{l}\text { Modelos de } \\
\text { procesamiento } \\
\text { de información } \\
\text { modulares } \\
\text { interactivos }\end{array}$ & 0 & \\
& & \\
$\begin{array}{l}\text { Distintos } \\
\text { desarrollos } \\
\text { en campos } \\
\text { a distintos } \\
\text { momentos: } \\
\text { percepción } \\
\text { del habla, } \\
\text { vocabulario, } \\
\text { gramática y } \\
\text { narrativa }\end{array}$ & & \\
\hline & & \\
\hline
\end{tabular}

También manifiestan abiertamente un conocimiento más sofisticado de algunos puntos en sus mismas respuestas a preguntas en un ejercicio de clase, como las siguientes: 
-Los modelos de procesamiento pueden ser modulares o interactivos. Los modulares muestran secuencias paso a paso con componentes separados $y$ los interactivos en un todo que relaciona todos los aspectos al tiempo.

-Los desarrollos del lenguaje se acompañan de desarrollos en la cognición; clasificar objetos se desarrolla con el vocabulario, la percepción con los objetos y con percibir el habla, y formar relaciones con la gramática y la narrativa.

- El desarrollo del lenguaje y de la cognición se alimentan uno con otro y no siempre uno está antes que el otro.

Los estudiantes también llegaron a dar cuenta de conceptos mostrados en los experimentos realizados por ellos mismos, por otros o los que se mostraron en documentales proyectados como parte de la clase. Al respecto, una nota del diario del profesor dice lo siguiente:

La observación del video 'The baby human' en una sesión de clase le sirvió a varios grupos para puntualizar detalles de su experimento, para acabar la formulación del problema (en los grupos que no lo han hecho todavia) y en general para comprender varios conceptos que se habian tratado en las sesiones magistrales. Hice entonces una ronda para indagar lo que sabian de los temas impartidos en las exposiciones magistrales y les mostré los ejemplos de experimentos que mejor los ilustraban en los videos para puntualizar mejor las explicaciones, $y$, al preguntarles de nuevo, las explicaciones de casi todos los estudiantes fueron muy buenas.

De otra parte, los estudiantes conocen el proceso de formulación de un problema y de su solución empírica. La mayoría de grupos pueden realizar el proceso completo de formulación de un problema, casi todos los grupos muestran poder formular un método coherente con la pregunta de investigación, y pueden planear e implementar la aplicación empírica del experimento sin mayores problemas, como lo indican las siguientes anotaciones del profesor.

A pesar de que algunos grupos se demoraron más que otros, todos los grupos finalmente pudieron realizar la formulación del problema de investigación. Al formular el método, la tarea resultaba relativamente más fácil para todos los grupos pero en dos grupos fue necesario llamarles la atención para que revisaran y acomodaran porque el método formulado no parecia acorde con el problema de investigación planteado. La aplicación del experimento no tuvo mayores problemas de planeación, aunque sí con la realización correcta o con una realización similar por parte de todos los grupos en la mitad de ellos.

Todos los grupos mostraron una mayor eficiencia al implementar la segunda unidad temática porque ya sabian el procedimiento a seguir desde el mismo momento en que recibieron los artículos y los temas de trabajo. De hecho, dos grupos de cada clase adelantaron su trabajo a un ritmo más rápido y sostenido que el resto.

En el mismo sentido, los estudiantes, especialmente los de grupos con un avance mayor en las sesiones de clase en el desarrollo de su experimento, formularon más preguntas y pidieron más ayuda al profesor sobre dudas conceptuales. La tabla 2 indica este comportamiento diferenciado de los grupos. 
TABLA 2. Niveles de AVANCE y SU RELACIÓN CON EL NÚMERO DE PREGUNTAS EN CADA SESIÓN Y CON LAS DUDAS QUE CADA GRUPO FORIULÓ AL PROFESOR POR INICIATIVA PROPIA

\begin{tabular}{|c|c|c|c|c|}
\hline Clase & Grupo & $\begin{array}{l}\text { Nivel de } \\
\text { avance }\end{array}$ & $\begin{array}{l}\text { Promedio de preguntas } \\
\text { / sesión }\end{array}$ & $\begin{array}{l}\text { Total dudas } \\
\text { conceptuales } \\
\text { formuladas al } \\
\text { profesor }\end{array}$ \\
\hline \multirow{5}{*}{$\begin{array}{l}\text { Diurna (n } \\
=14 \text { ) }\end{array}$} & Grupo 1 & Esperado & 3 & 13 \\
\hline & Grupo 2 & Esperado & 3 & 6 \\
\hline & Grupo 3 & Lento & 1 & 2 \\
\hline & Grupo 4 & Avanzado & 5 & 12 \\
\hline & Grupo 5 & Atrasado & 0 & 2 \\
\hline \multirow{6}{*}{$\begin{array}{l}\text { Nocturna } \\
(n=16)\end{array}$} & Grupo 1 & Avanzado & 5 & 9 \\
\hline & Grupo 2 & Esperado & 4 & 5 \\
\hline & Grupo 3 & Esperado & 3 & 5 \\
\hline & Grupo 4 & Lento & 1 & 2 \\
\hline & Grupo 5 & Atrasado & 1 & 2 \\
\hline & Grupo 6 & Avanzado & 7 & 9 \\
\hline
\end{tabular}

En otro plano, los estudiantes consolidaron parcialmente su conocimiento sobre convenciones propias de los reportes escritos de investigación. A pesar de la estrategia implementada, la mayoria de estudiantes siguieron teniendo dudas o no comprendieron convenciones propias de los reportes de investigación, como se evidencia en la tabla 3.

TABLA 3. LOGRO DE LOS ESTUdIANTES EN CADA UNO DE LOS ASPECTOS DEL INFORME FINAL DE LABORATORIO EN LAS DOS UNIDADES DIDÁCTICAS

\begin{tabular}{llll}
$\begin{array}{l}\text { Aspecto del } \\
\text { informe de } \\
\text { laboratorio }\end{array}$ & $\begin{array}{l}\text { Grupos de } \\
\text { estudiantes } \\
\text { que lo tienen } \\
\text { completamente } \\
\text { bien }\end{array}$ & $\begin{array}{l}\text { Grupos de } \\
\text { estudiantes } \\
\text { que lo tienen } \\
\text { parcialmente bien }\end{array}$ & $\begin{array}{l}\text { Grupos de } \\
\text { estudiantes } \\
\text { que lo tienen } \\
\text { incorrectamente }\end{array}$ \\
\hline Forma y formato & 1 & 1 & 9 \\
\hline $\begin{array}{l}\text { Contenido de la } \\
\text { introducción }\end{array}$ & 4 & 2 & 5 \\
\hline $\begin{array}{l}\text { Contenido del } \\
\text { método }\end{array}$ & 3 & 4 & 4 \\
\hline $\begin{array}{l}\text { Contenido de los } \\
\text { resultados }\end{array}$ & 1 & 2 & 8 \\
\hline
\end{tabular}

Además de lo anterior, los apuntes del profesor muestran que los estudiantes siguieron presentando sus informes en otros formatos diferentes al dado (uno próximo a las normas de la apa), fueron realizados sin suficiente tiempo, no aprovechaban todos los apuntes que habian tomado en la elaboración del experimento, y no reconocían aún el tipo de contenido que debía ir en cada sección. Los apuntes son los siguientes: 
No se encontraron grandes avances en el proceso de composición del informe escrito. Es posible que se trate de una tarea en si misma bastante compleja y que necesita, probablemente, más refuerzo en la formación de estos estudiantes.

La mayoria de informes fueron devueltos para ser revisados por los grupos porque estaban todavia en un nivel muy deficiente de elaboración. A la siguiente semana se realizó con cada grupo de estudiantes una revisión de su informe durante la sesión de clase. A pesar de las retroalimentaciones, era común todavía en algunos que no siguieran las instrucciones de inicio en el formato, que mezclaran partes metodológicas en la introducción, que la metodología no fuera totalmente explicita o que los resultados solamente tuvieran un relato anecdótico y no registraran el cuerpo de evidencia obtenido mediante tablas, gráficos o muestras de trabajo de los sujetos participantes.

Me llamó particularmente la atención que en dos grupos de los once, tuve que mostrarles que los apuntes de lo que hacian en el procedimiento de desarrollo del laboratorio contenía información que necesitaban para complementar su informe. Este punto necesita revisión profunda porque es todavia dificil hacer que los estudiantes comprendan la lógica y la estructura del informe de laboratorio o que reporta resultados de investigación. Es posible que deba trabajarse más en las sesiones temáticas en revisar articulos de investigación $y$ sus características, porque dos estudiantes me preguntaron por dudas específicas sobre dónde encontraban cierta información en los artículos de investigación que habian conseguido como complemento a los que ya tenian.

A pesar de saber los pasos generales para la formulación, elaboración y presentación del experimento, no existe evidencia de que los estudiantes razonen en términos de problema de investigación. En otras áreas diferentes al desarrollo del experimento en las unidades temáticas presentadas, muestran una tendencia a usar un procedimiento para soluciones de momento y no aplicadas a otras situaciones similares.

Varios estudiantes no evidenciaron aún comprensión de la lógica de avance del conocimiento a juzgar por las preguntas que formularon en exposiciones magistrales como las siguientes: “¿Finalmente, qué de todo eso es cierto?, porque si son teorías algunas deben ser ciertas, ¿no?" O "Profesor, cuando están esos modelos, ¿qué es lo que se averigua o qué termina siendo lo que sí es cierto? o ¿nada es cierto?, ¿que han averiguado?" En el mismo sentido, los estudiantes formularon preguntas sobre el desarrollo de su laboratorio, casi siempre orientadas a aspectos puntuales del procedimiento a desarrollar. Pocas veces orientadas a la coherencia general del experimento con el problema de investigación que habian formulado o con la organización general del informe de laboratorio, como se muestra en la tabla 4.

TABLA 4. Aspectos sobre los que Versan las PREGUNTAS ESPONTÁNEAS DE LOS ESTUDIANTES EN LAS SESIONES DE LABORATORIO

Aspecto aludido por
las preguntas de los
estudiantes

Deducción para llegar a la pregunta

Formulación de método

\section{Aspectos tenidos en cuenta en la formulación del método}
Aspectos del plan de desarrollo de trabajo empírico

Instrucciones para el desarrollo del informe final

\section{Coherencia entre el problema, la pregunta, el experimento formulado y los resultados}




\section{Resumen de hallazgos}

Los estudiantes avanzaron en conocimiento de conceptos de la asignatura. Mostraron un dominio esperado en ellos al finalizar las dos unidades temáticas y podían dar cuenta de este dominio al preguntárseles oralmente o por escrito sobre los conceptos que usaron.

Dichos estudiantes también avanzaron en el conocimiento de cómo formular un problema de investigación. Sin embargo, dicho avance es aún limitado, al parecer, a realizar procedimientos, porque se usa sólo como fórmula repetida pero no como un modelo flexible que se acomoda a la realidad para poderla interpretar.

Los alumnos participantes de esta experiencia no mostraron gran avance en su conocimiento sobre la presentación de reportes de investigación en psicología. Todavía tienen confusiones en el uso de formatos y en el tipo de información que debe colocarse en cada sección de un informe de investigación experimental.

\section{Discusión}

En la sección anterior se expusieron los resultados de esta experiencia. Esta sección está dividida en dos apartados: uno sobre el proceso de aprendizaje observado en los estudiantes y otro sobre limitaciones y aspectos a mejorar en el estudio. Ambas se desarrollan a continuación.

\subsection{Aprendizaje basado en reproducir realidades y conocimientos convencionales de la disciplina}

El avance de los estudiantes en conocimientos básicos de la asignatura y en la metodología de indagación investigativa y experimental en su campo, es una evidencia a favor de los modelos de aprendizaje basados en problemas en el contexto universitario. Diversos modelos como el Aprendizaje Basado en Problemas (Duenas, 2001; Echavarria, 2010; Gorbaneff \& Cancino, 2009; Kindler et.al., 2009; Kreiner, 2009; Newton \& Wood, 2009; Restrepo Gomez, 2005), el diseño de ambientes de aprendizaje con análisis de tareas (Flórez, Arias \& Guzmán, 2006; Larreamendy, 2003) y la Enseñanza para la Comprensión (Perkins \& Blythe, 1994;
Mejía \& Flórez, 2011) coinciden en que no es suficiente con dictar contenido de modo magistral o a modo de conferencia y es necesario que el estudiante aprenda también maneras de plantear problemas, procedimientos y exploración de vías de solución a los mismos para que avancen en el conocimiento tanto de habilidades como de conceptos propios de las disciplinas de estudio en el ámbito universitario (Etkina, Van Heuvelen, White-Bramia, Brookes, Gentile, Murhty, Rosengrant \& Warren, 2006).

Esto no quiere decir que se abandone la idea de un papel importante de instrucción y enseñanza de parte del profesor. Lo que más bien se quiere plantear es que las conferencias magistrales, las exposiciones y la demostración con ejemplos, siguen siendo estrategias válidas y efectivas para el logro del aprendizaje de los estudiantes universitarios (Camargo \& Hederich, 2007), pero dichas estrategias parecen funcionar mejor cuando logran insertarse en el marco de una actividad mayor que les confiera sentido, como un proyecto de indagación que también permita a los estudiantes ejercitar sus habilidades y realizar sus propias reflexiones y sus propias búsquedas dentro del límite de una pauta temática dada por el profesor (Ministerio de Educacion Nacional, Colombia, 2009).

Una pregunta muy importante en este punto y ante los resultados de esta experiencia es si lo que han aprendido los estudiantes en el curso puede hacer que piensen diferente. Se espera que los cursos universitarios de pregrado le permitan al estudiante de psicología aprender a formular problemas y a indagar en su área de conocimiento a través de las evidencias y los reportes de literatura científica (Carlino, 2005; Flórez \& Gutiérrez, 2009). Sin embargo, las evidencia de este curso y otras experiencias similares y de estudios en educación superior, muestran que con frecuencia este logro no se alcanza por limitaciones de tiempo o de funcionamiento institucional $\mathrm{y}$, se propone como alternativa, el trabajo con los estudiantes como participantes de niveles básicos en investigaciones reales llevadas a cabo por investigadores formados.

La evidencia de este estudio y la de algunos otros similares muestra que en las actividades de investigación formativa o de reproducción de actividades de investigación en pequeña escala, los estudiantes llegan a aprender, siempre que al reducir la escala de trabajo para 
que sea manejable por el estudiante no sacrifique la complejidad del proceso (Hernández, 2003; Flórez, Castro \& Arias, 2009; Restrepo Gomez, 2003). Por lo tanto, las tareas, situaciones y actividades con los estudiantes deben ser en una pequeña escala manejable pero que conserve y muestre a estos la complejidad del campo de conocimiento que abordan (Flórez, Castro \& Arias, 2009). De todos modos se reconoce que por ser este un estudio de un nivel apenas exploratorio, es necesario tener evidencia más fuertes y generar datos más robustos y fiables que confirmen o refuten las tendencias que se observan en esta experiencia, que tiene validez dentro de su contexto, pero no necesariamente en otro con otras condiciones.

\subsection{Limitaciones encontradas y aspectos que pueden mejorar}

Así como se han señalado algunos alcances, es necesario puntualizar algunas limitaciones de esta experiencia. La primera es de tipo curricular: la mayoría de planes de estudio, incluido el del programa de psicología, en el cual se inscribe la asignatura objeto de este estudio, suelen dividir muchas de las actividades que consideran más importantes en asignaturas completamente separadas en un solo semestre. Las principales habilidades de muchos estudiantes universitarios realmente consolidan su aprendizaje a lo largo de todo su paso por el programa, por lo cual es importante observar la necesidad de planear líneas de formación y trabajo más continuas con los estudiantes (Rodríguez, 2007).

De la mano con la limitación anterior, está la necesidad de conocimiento uso y familiarización de los estudiantes con la literatura especializada de su área de estudio. Gran parte de la formación de los estudiantes de pregrado está relegada a las cátedras y no se atiende muchas veces con suficiencia si los estudiantes reconocen elementos importantes de un artículo, un capítulo o un libro propios de su disciplina (Carlino, 2005; Flórez \& Gutiérrez, 2009). Esta es un área de trabajo continua que debe ser fortalecida en todas las formaciones de base cientifica e investigativa, entre las cuales se incluye la psicología.

Otra limitación que debe advertirse en el desarrollo de experiencias como ésta, es el proceso de expectativa del docente y de los estudiantes (Rogers, 1988). Del docente en el contexto universitario se esperan algunas caracteristicas, habilidades y comportamientos, lo mismo que de los estudiantes. En el caso de los estudiantes, estos parecen en muchos casos (por su historia de formación previa en todo el sistema educativo) estar acostumbrados a trabajar por una calificación o un resultado que permita la promoción y con menos frecuencia por un aprendizaje significativo e importante para la vida posterior (Wells, 1986). Es necesario pensar en estrategias que muestren al estudiante alternativas a esta visión y le manifiesten la importancia del logro en el aprendizaje por sí mismo y no sólo como el camino a una calificación (este problema claramente trasciende los limites de un estudio como éste pero que afecta sus resultados y su alcance).

Es necesario también crear mecanismos que fortalezcan más el dialogo y la retroalimentación continua entre los conceptos o el contenido impartido de manera magistral, y los procedimientos que se dan a realizar a los estudiantes (Etkina et. al, 2006). Esta experiencia, como muchas otras, no resuelve del todo este problema y pude ser necesario seguir trabajando en él.

Finalmente, se sugiere realizar acompañamiento externo y observar los resultados de estudiantes en evaluaciones externas y en rendimientos futuros para confirmar los hallazgos y las hipótesis que este estudio plantea (ICFES, 2010). 


\section{Referencias}

1. Camargo, A. \& Hederich, C. (2007). El estilo de comunicación y su presencia en el aula de clase. Folios, 26, 3-12.

2. Carlino, P. (2005). Escribir, leer y aprender en la universidad. México: Fondo de Cultura Económica.

3. Dueñas, V. H. (2001). El aprendizaje basado en problemas como enfoque pedagógico de la educación en salud. Colombia Médica, 32(4), 189-196.

4. Echavarria, M. V. (2010). Problem - based learning application in engineering. Revista EIA (Escuela de Ingeniería de Antioquia, Medellín, Colombia), 14, 85-95.

5. Elliott, J. (2000). El cambio educativo desde la investigación - acción. Madrid: Morata.

6. Etkina, E., Van Heuvelen, A., White-Bramia, S., Brookes, D., Gentile, M., Murhty, S., Rosengrant, D. \& Warren, A. (2006). Scientific abilities and their assessment. Physical Review Special Topics - Physics Education Research, $2,1-15$.

7. Flórez, R., Arias, N. \& Guzmán, R. J. (2006). El aprendizaje en la escuela: el lugar de la lectura y la escritura. Educación y Educadores, 9(1), 117-133.

8. Flórez, R., Castro, J. \& Arias, N. (2009). Comunicación, lenguaje y educación. Una mirada desde las teorías de la complejidad. Folios, 30, 25-38.

9. Flórez, R. \& Gutiérrez, M. J. (2009). Alfabetización académica: una propuesta para la formación de docentes universitarios. Bogotá: Universidad Nacional de Colombia.

10. Gorbaneff, Y. \& Cancino, A. (2009). Mapa conceptual para el aprendizaje basado en problemas. Estudios Gerenciales, 25(110), $111-124$
11. Hernández, C. A. (2003). Investigación e investigación formativa. Nómadas, 18, 183-193.

12. Instituto Colombiano de Fomento a la Educacion Superior, ICFES (2010). Colombia en PISA 2009. Síntesis de resultados. Bogotá: ICFES.

13. Kindler, P., Grant, C., Kulla, S., Poole, G. \& Godolphin, W. (2009). Difficult incidents and tutor interventions in problema - based learning tutorials. Medical Education, 43, 866-873.

14. Kreiner, D. S. (2009). Problem - based group activities for teaching sensation and perception. Teaching of Psychology: Methods and Techniques, 36, 253-256.

15. Larreamendy, J. F. (2003). Ciencia cognitiva y educación: más allá de la falacia de la aplicación. Diálogos, Discusiones en la Psicología Contemporánea, 2, 139-151.

16. Mejía, C. P. \& Flórez, R. (2011). El marco de la enseñanza para la comprensión aplicado al aprendizaje del concepto de campo eléctrico en estudiantes de ingeniería de sistemas. Tesis inédita presentada por la primera autora bajo dirección de la segunda, para optar al título de Magister en Educacion - Comunicación y Educacion, Maestría en Educacion, Facultad de Ciencias Humanas, Universidad Nacional de Colombia.

17. Ministerio de Educacion Nacional, Colombia, (2009). Desarrollo infantil y competencias en la primera infancia. Bogotá: Ministerio de Educación Nacional, Doc. No. 10.

18. Newton, C. \& Wood, V. (2009). Reflections on facilitating an interprofessional problem - based learning module. Journal of Interprofessional Care, 23(6), 672-675.

19. Perkins, D. \& Blythe, T. (1994). Putting understanding up front. Educational Leadership, 51(5): 4-7.

20. Restrepo Gómez, B. (2003). Conceptos y aplicaciones de la investigación formativa y criterios para evaluar la investigación estricto sentido. En Consejo Nacional de Acreditación, 
CNA (ed.), Educacion Superior, Calidad y Acreditación, Tomo 1 (pp. 8 -13). Bogotá: CNA.

21. Restrepo Gómez, B. (2005). Aprendizaje basado en problemas (ABP): una innovación didáctica para la enseñanza universitaria. Educación y Educadores, 8, 9-19.

22. Rodríguez, R. (2007). Currículo y educación superior. Presentación en la Línea Comunicación y Educacion, Maestría en Educacion, Universidad Nacional de Colombia. Video inédito en formato DVD.

23. Rogers, C. (1988). Psicología social de la enseñanza. Madrid: Visor.

24. Rojas, S. P. \& Flórez, R. (2010). Evaluación de un plan de formación para docentes no profesionales en educación: pedagogía de la lectura y la escritura. Educacion y Educadores, 13(3), 377-396.

25. Stenhouse, L. (1980). Curriculum research and development in action. Chicago, IL: Heinemann Educational.

26. Stenhouse, L. (1981). What counts as research? British Journal of Educational Studies, 29(2), 103-114.

27. Vygotski, L. S. (1978). El desarrollo de los procesos psicológicos superiores. Barcelona: Crítica.

28. Wells, G. (1986). The Meaning Makers. Londres: Hodder \& Stoughton. 